\title{
Non-periodic driving of coupled oscillators: a spherical swing
}

\author{
J.R. Sanmartín ${ }^{\mathrm{a}}$, O. López-Rebollal ${ }^{\mathrm{a}}$ and N. de Paola ${ }^{\mathrm{b}, 1}$ \\ "ETS Ingenieros Aeronáuticos, Universidad Politécnica, 28040 Madrid, Spain \\ ${ }^{\mathrm{b}}$ Fluid Mechanics Laboratory, Massachusetts Institute of Technology, Cambridge, MA 02139, USA
}

Received 16 November 1992

Revised manuscript received 7 May 1993

Accepted 28 May 1993

Communicated by F.H. Busse

\begin{abstract}
Nonlinearly coupled, damped oscillators at 1:1 frequency ratio, one oscillator being driven coherently for efficient excitation, are exemplified by a spherical swing with some phase-mismatch between drive and response. For certain damping range, excitation is found to succeed if it lags behind, but to produce a chaotic attractor if it leads the response. Although a period-doubling sequence, for damping increasing, leads to the attractor, this is actually born as a hard (as regards amplitude) bifurcation at a zero growth-rate parametric line; as damping decreases, an unstable fixed point crosses an invariant plane to enter as saddle-focus a phase-space domain of physical solutions. A second hard bifurcation occurs at the zero mismatch line, the saddle-focus leaving that domain. Times on the attractor diverge when approaching either line, leading to exactly one-dimensional and noninvertible limit maps, which are analytically determined.
\end{abstract}

\section{Introduction}

A second-order nonlinear oscillator under harmonic excitation is an usual model for deterministic chaos in open, dissipative systems. Classicals such as the pendulum [1], Van der Pol [2], and Duffing [3] equations show chaotic behavior. For nonlinear oscillators, however, nonautonomous, harmonic excitation is just one particular model; basic to it is the fact that the oscillator frequency is amplitude dependent, the response thus getting out of phase with sine-time parametric or forcing laws. An opposite model would keep driving at a definite phase with the response, feeding energy coherently, by way of feedback, to achieve efficient excitation. In this paper we explore whether, and how, a phase mismatch might make the response chaotic.

\footnotetext{
${ }^{1}$ Present address: Biomedical Engineering Department, Northwestern University, Evanston, IL 60208-3107, USA.
}

Chaos is precluded if the equation for the oscillations, now autonomous, is second order, as in a swing. To get a complex dynamics on a low-dimensional manifold, we thus consider two linear oscillators under weak (nonlinear) coupling, damping, and coherent, non-periodic excitation on one of them. We then expect to end up with 3 first-order differential equations for two amplitudes and a phase-difference (the phase of each oscillation being unimportant by itself); they might thus resemble equations for resonant 3-wave coupling with two waves equally damped and a third one excited. This reduced case, studied in plasma physics [4-6], only involves two wave amplitudes; three-wave interaction is discussed by Guckenheimer and Mahalov [7]. There are old and new examples of coherent excitation of physical oscillators [8]; driving economical cycles might be a social application of interest.

In the present work we consider oscillators 
with a $1: 1$ frequency ratio arising in a symmetrical system, the "spherical swing". The weak forcing of a spherical pendulum along a given horizontal axis following the usual sine-time law was studied by Miles, [9], who found that chaos might set in no matter how weak the excitation; attempts at simple, experimental checks have provided partial agreement with theory [10]. Our problem differs essentially from Miles' in that, as in a swing, we take forcing coherent with the response along its own direction; because of the nonlinearity, it cannot then keep phase with the response along the perpendicular horizontal direction. This non-periodic drive leads to a 3rdorder system of equations, as against a 4th-order system for the usual, harmonic drive [9].

In section 2 we obtain model equations with phase-mismatch and damping parameters; we avoid the formalism of feedback [11]. Section 3 gives simple analytical and numerical results, showing that a chaotic attractor does develop within some definite parametric domain. In section 4 we show that this attractor is born as a hard (as regards amplitude) bifurcation at two parametric lines, when a saddle-focus enters a phase-space domain of physical solutions; this may result in exactly one-dimensional and noninvertible return maps. In sections 5 and 6 we analytically derive such maps, which are bimodal near the corner of the lines. Results are summarized in section 7 .

\section{The model equations}

Consider a spherical pendulum driven along a horizontal direction $x$ (say by motion of its point of support), with a force per unit mass of bob

$f_{x} \equiv \epsilon g \sin \omega t$,

$\epsilon$ being small and $\omega$ close to the natural frequency $\omega_{0} \equiv(g / R)^{1 / 2} ; R$ is the radius and $\nu \equiv$ $\left(\omega^{2}-\omega_{0}^{2}\right) / \omega^{2} \epsilon^{2 / 3}$ is of order unity [9]. No matter how small $\varepsilon$, there is a time-scale for nonlinear coupling of oscillations along the horizontal axes $x$ and $y$. Introducing a linear drag (per unit mass) $-\alpha \epsilon^{2 / 3} \omega_{0} \times$ velocity, and a slow scale $\tau \equiv$ $\frac{1}{2} \epsilon^{2 / 3} \omega_{0} t$, Miles posed the solution as

$\frac{x}{\epsilon^{1 / 3} R}=p_{1}(\tau) \cos \omega t+q_{1}(\tau) \sin \omega t$,

$\frac{y}{\epsilon^{1 / 3} R}=p_{2}(\tau) \cos \omega t+q_{2}(\tau) \sin \omega t$,

and found averaged equations for $p_{1}, q_{1}, p_{2}$ and $q_{2}$, involving the parameters $\alpha$ and $\nu$. Rewriting $x$ and $y$ as almost sinusoidal,

$\frac{x}{\epsilon^{1 / 3} R}=a(\tau) \cos [\omega t+\phi(\tau)]$,

$\frac{y}{\epsilon^{1 / 3} R}=b(\tau) \cos [\omega t+\psi(\tau)]$,

we can obtain the equations for the slow evolution of amplitudes $a, b$, and phases $\phi, \psi$, directly from system $(2.13 \mathrm{a}-\mathrm{d})$ in ref. [9],

$\dot{a}=-\alpha a-\frac{3}{8} a b^{2} \sin 2(\phi-\psi)-\cos \phi$,

$\dot{\phi}=\nu-\frac{1}{8} a^{2}+\frac{1}{4} b^{2}\left[1-\frac{3}{2} \cos 2(\phi-\psi)\right]+\frac{\sin \phi}{a}$,

$\dot{b}=-\alpha b+\frac{3}{8} b a^{2} \sin 2(\phi-\psi)$,

$\dot{\psi}=\nu-\frac{1}{8} b^{2}+\frac{1}{4} a^{2}\left[1-\frac{3}{2} \cos 2(\phi-\psi)\right]$.

The flow divergence of eqs. $(2 \mathrm{a}-\mathrm{d})$ is constant and negative:

$\frac{1}{a} \frac{\partial}{\partial a}(a \dot{a})+\frac{\partial \dot{\phi}}{\partial \phi}+\frac{1}{b} \frac{\partial}{\partial b}(b \dot{b})+\frac{\partial \dot{\psi}}{\partial \psi}=-4 \alpha$,

as given by Miles for his equivalent system.

Here we consider a different driving force,

$f_{x}=-\epsilon g \rho \sin [\omega t+\phi(\tau)+\sigma]$,

coherent with the response. Since we have $-\dot{x} \propto a \sin (\omega t+\phi)$ as $\epsilon \rightarrow 0$, we may write $f_{x} \propto(\rho / a)(\dot{x} \cos \sigma-\omega x \sin \sigma) ;$ for $\sigma=0$, the power, $f_{x} \dot{x}$, would be positive throughout. Allowing, however, for a constant phase-mismatch $\sigma$, the range of interest for excitation would 
clearly be $-\frac{1}{2} \pi<\sigma<\frac{1}{2} \pi$. We also allow a slow adjustment of drive strength through a factor $\rho(a)>0$ dependent on the excited amplitude. Only the last term in eqs. (2a), (2b) need be modified. One may find the new equations by comparing $-\rho \sin (\omega t+\varphi+\sigma)$ in $\left(1^{\prime}\right)$ with $\sin \omega t$ in (1), both written in terms of $\sin (\omega t+\varphi)$ and $\cos (\omega t+\varphi)$,

$\dot{a}=-\alpha a-\frac{3}{8} a b^{2} \sin 2(\phi-\psi)+\rho \cos \sigma$, $\dot{\phi}=\nu-\frac{1}{8} a^{2}+\frac{1}{4} b^{2}\left[1-\frac{3}{2} \cos 2(\phi-\psi)\right]+\frac{\rho}{a} \sin \sigma$.

We could now remove $\nu$ from the problem by absorbing it into both $\phi$ and $\psi(\phi, \psi \rightarrow \phi+$ $\nu \tau, \psi+\nu \tau)$ : for coherent driving there is no a priori frequency $\omega$. Actually, the difference between eqs. $\left(2 b^{\prime}\right)$ and $(2 d)$, together with $\left(2 a^{\prime}\right)$ and (2c), yields equations for $a, b$, and $\gamma \equiv 2(\phi-$ $\psi$ ) in a reduced three-dimensional phase-space, $\nu$ just dropping off. Though not essential to chaos, we take $\rho(a)$ such as to have an uniform flow divergence, for simplicity, $\rho=k a+k^{\prime} / a, k$ and $k^{\prime}$ being arbitrary constants. For $k=0$ the system can be integrated once [12]; here we consider the next simple case, $k^{\prime}=0$, and set $k=1$ without loss of generality. Note that for the amplitude $a$ (but not $b$ ) to grow indefinitely as desired, $\rho$ should indeed increase with ' $a$ ' as fast as the drag, $\alpha a$; also, $\rho(a) \propto a$ leads to a natural, simple form for $f_{x}$ when $\sigma=0, f_{x} \propto \dot{x}$.

Rescaling time and energy, $2 \tau \rightarrow \tau, \frac{3}{8} a^{2} \equiv A$, $\frac{3}{8} b^{2} \equiv B$, we finally obtain the equations for our model,

$\dot{A}=(\cos \sigma-\alpha) A-A B \sin \gamma$,

$\dot{B}=-\alpha B+A B \sin \gamma$,

$\dot{\gamma}=\sin \sigma-(A-B)(1-\cos \gamma)$.

This system, aside from different meanings for growth rate and mismatch, is similar to a system for reduced 2-wave cubic coupling derived from the nonlinear Schrödinger equation in ref. [6]; eqs. $(6 a-c)$ there, when conveniently rear- ranged, differ only in the phase-difference equation, which would read

$\dot{\gamma}=\sin \sigma-(A-B)(1-\cos \gamma)-\frac{1}{2} B$.

We shall comment on this difference in section 4.

\section{Attractors of the model}

We first go over several features of system $(3 a-c)$ that are readily determined analytically. (i) The flow has a divergence, $\partial \dot{A} / \partial A+\partial \dot{B} /$ $\partial B+\partial \dot{\gamma} / \partial \gamma=\cos \sigma-2 \alpha$, uniform throughout phase-space, which is a $1 \mathrm{D}$ torus $(\gamma) \times$ a plane $(A, B)$. (ii) The surfaces $A=0$ and $B=0$ are invariant, $A$ and $B$ keeping positive and bounded at any finite time if initially positive. (iii) The normal rest position, $A=B=0$, is a stationary equilibrium, the equation for $\gamma$ then being physically irrelevant. (iv) There is, in addition, one stationary point $\mathrm{P}$ given by the equations

$$
\begin{aligned}
& A_{\mathrm{P}}=\frac{\alpha}{\sin \gamma_{\mathrm{P}}}, \quad B_{\mathrm{P}}=\frac{\cos \sigma-\alpha}{\sin \gamma_{\mathrm{P}}}, \\
& \frac{1-\cos \gamma_{\mathrm{P}}}{\sin \gamma_{\mathrm{P}}} \equiv \tan \frac{1}{2} \gamma_{\mathrm{P}}=\frac{\sin \sigma}{2 \alpha-\cos \sigma} ;
\end{aligned}
$$

$A$ and $B$ are positive for either $\sigma<0, \alpha<\frac{1}{2} \cos \sigma$ or $\sigma>0, \frac{1}{2} \cos \sigma<\alpha<\cos \sigma$ (fig. 1). (v) The characteristic equation for $P$ is

$$
\begin{aligned}
s^{3} & +(2 \alpha-\cos \sigma) s^{2}+\sin ^{2} \sigma \frac{\alpha(\cos \sigma-\alpha)}{(2 \alpha-\cos \sigma)^{2}} s \\
& +\frac{\alpha}{2} \frac{\cos \sigma-\alpha}{2 \alpha-\cos \sigma}\left(1-4 \alpha \cos \sigma+4 \alpha^{2}\right)=0 .
\end{aligned}
$$

In the $\sigma>0$ domain, there is a negative root and a Hopf bifurcation at the line $\alpha=\frac{1}{2}(\cos \sigma+$ $\sin \sigma)$, the fixed point being stable below it; in the $\sigma<0$ domain the real root is positive and $\mathrm{P}$ is always unstable.

We next show that chaos will not set in outside the parameter domain defined by $\frac{1}{2} \cos \sigma<\alpha<$ $\cos \sigma, \sigma>0$ (fig. 1): For $\alpha>\cos \sigma$, the equilib- 


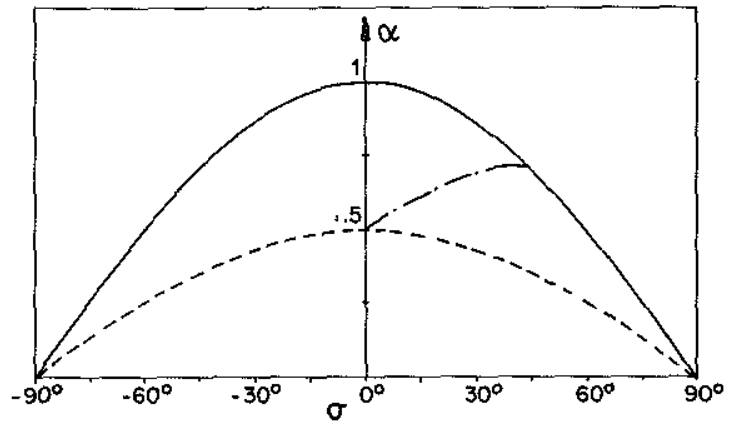

Fig. 1. Attractors vs damping coefficient $\alpha$ and phase mismatch $\sigma ; A=B=0$ (bob at rest) above $\alpha=\cos \sigma$ (-); $A \rightarrow \infty, B \rightarrow \infty$ (unbounded nonplanar motion) below $\alpha=$ $\frac{1}{2} \cos \sigma(--) ; A \rightarrow \infty, B \rightarrow 0$ (unbounded planar motion) for $\frac{1}{2} \cos \sigma<\alpha<\cos \sigma, \sigma<0$. For $\frac{1}{2} \cos \sigma<\alpha<\cos \sigma, \sigma>0$, equilibrium point below $\alpha=\frac{1}{2} \cos \sigma+\frac{1}{2} \sin \sigma(-\cdot)$, limit cycles or chaos above.

rium $A=B=0$ is globally stable; that equilibrium is clearly unstable for $\alpha<\cos \sigma$. For $\alpha<$ $\frac{1}{2} \cos \sigma$, the flow divergence is positive, any initial phase volume growing monotonically at the exponential constant rate $\cos \sigma-2 \alpha$; since surfaces of constant energy $(A+B)$ are nested with volume $\propto$ (energy $)^{2}$, energy will also grow without bound along trajectories starting at arbitrary points, except possibly for a set of zero measure: for negative $\sigma$, that set includes point $\mathrm{P}$ above the line $\alpha=\frac{1}{2}(\cos \sigma+\sin \sigma)$, and point $\mathbf{P}$ and its stable two-dimensional manifold below it. For $\frac{1}{2} \cos \sigma<\alpha<\cos \sigma$ and $\sigma<0$, the limit set $A \rightarrow \infty, \mathrm{B} \rightarrow 0,0 \leq y<2 \pi(\bmod 2 \pi)$, is an attractor; note that phase volumes are now contracting. If $A$ and $1 / B$ are large, eq. (3c) gives a monotonous decrease for $\gamma$,

$\dot{\gamma} \simeq-[|\sin \sigma|+A(1-\cos \gamma)]$

$A$ (and $B$ ) changing little within the short period from $\gamma=2 \pi$ to $\gamma=0, \Delta \tau \simeq 2 \pi /(2|\sin \sigma| A)^{1 / 2}$. From (3a) we have

$$
\dot{A} / A=\cos \sigma-\alpha>0 \quad(A \rightarrow \infty \text { as } \tau \rightarrow \infty) .
$$

Also, using eqs. (3b) and (5) to average $\dot{B} / B$ over the period $\Delta \tau$, we obtain
$\frac{1}{\Delta \tau} \ln \frac{B(0)}{B(2 \pi)}+\alpha=\int_{0}^{2 \pi} \frac{A \sin \gamma \mathrm{d} \gamma}{-\dot{\gamma} \Delta \tau}=\frac{1}{\Delta \tau} \ln \frac{A(0)}{A(2 \pi)}$,

yielding, from (6), $\dot{B} / B \simeq B \simeq \cos \sigma-2 \alpha<0$ $(B \rightarrow 0$ as $\tau \rightarrow \infty)$.

Consider therefore the domain $\frac{1}{2} \cos \sigma<\alpha<$ $\cos \sigma, \sigma>0$. Below the line $\alpha=\frac{1}{2}(\cos \sigma+\sin \sigma)$, the (stable) fixed point $\mathrm{P}$ is a global attractor. The Hopf bifurcation at that line is found to be supercritical [13], but, beyond it, volume contraction precludes secondary bifurcation of the ensuing limit cycle to a 2-torus. Numerical results show, instead, the familiar period-doubling sequence. Figure 2 shows orbits for $\sigma=3.75^{\circ}$, projected onto the $A-B$ plane in the interval $1000<\tau<4000$, to exhibit long-time attractors:

(a) a 2-cycle, (b) a 16-cycle, (c) an apparently aperiodic attractor; initial values were $A_{0}=B_{0}=$ $\frac{3}{2}, \gamma_{0}=\frac{1}{2} \pi$. For $\sigma=3.75^{\circ}$ the simple limit cycle is born at $\alpha_{1}=0.5316 \ldots$ It loses stability at $\alpha_{2} \simeq$ $0.696 ; 4-, 8-$, and 16-cycles are born at $\alpha_{4} \simeq$ $0.7740, \alpha_{8} \simeq 0.7922$, and $\alpha_{16} \simeq 0.7960$, respectively. The ratios

$\frac{\alpha_{4}-\alpha_{2}}{\alpha_{8}-\alpha_{4}} \simeq 4.3 \pm 0.15, \frac{\alpha_{8}-\alpha_{4}}{\alpha_{16}-\alpha_{8}} \simeq 4.8 \pm 0.3$,

suggest that the sequence approaches Feigenbaum's constant, 4.669 ...

Figure 3 i plots each maximum in $A$ along the orbit against the previous one: the points fall in a smooth arc with a simple extremum, a nearly one-dimensional non-invertible map, $A_{k+1}\left(A_{k}\right)$, the Cantor structure being here very weak. The exponent of the parabola at the extremum, as simply estimated from the graph, differs by less than $5 \%$ from the value 2 appropriate for Feigenbaum's constant. The fixed point at the bisector is unstable. The multiple fixed points for doubling, $A_{k+2}\left(A_{k}\right)$, quadrupling, $A_{k+4}\left(A_{k}\right)$, etc., iterates, also prove to be unstable (figs. 3ii, iii).

The attractor exhibits sensitivity to initial conditions. The distance $D$ between trajectories starting at close points, one being the point at 

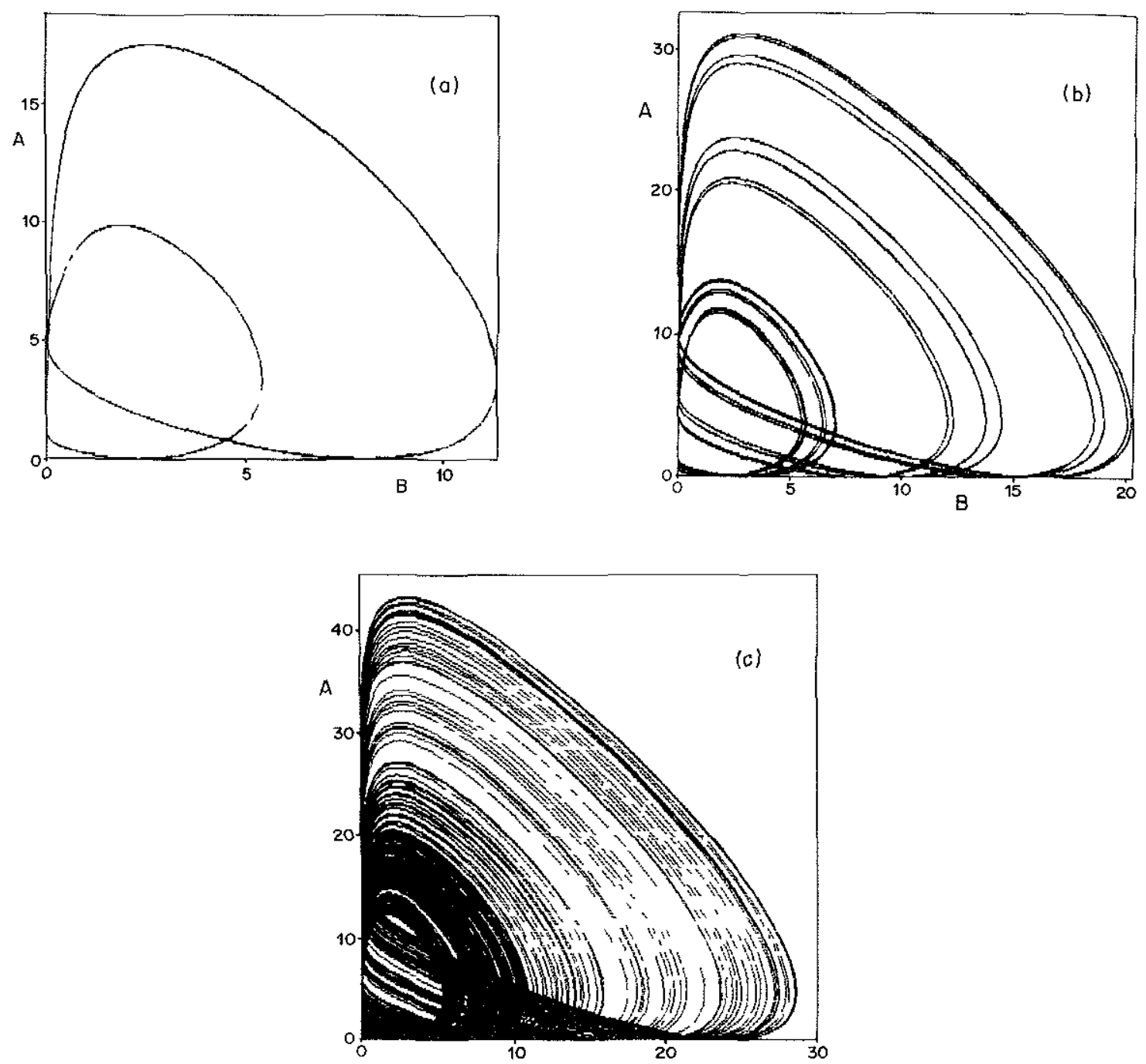

Fig 2. Orbits projected onto $A-B$ plane at large times, for $\sigma=375^{\circ}$ and (a) $\alpha=075$, (b) $\alpha=079675$, (c) $\alpha=082$

$\tau=2000$ in the orbit of fig. $2 \mathrm{c}$, diverges exponentially in the mean before saturating at about the size of the attractor ( $A$ maximum $\sim 44$, fig. $3 \mathrm{i}$ ): the largest Lyapunov exponent, $\lambda_{1} \equiv d \ln D / \mathrm{d} \tau \simeq$ $1.95 \times 10^{-2}$, is constant and positive (fig. 4). Using an estimate reasonably contrasted for uniform volume-contraction [14] we get a fractal dimension for the attractor,

Dimension $=2+\frac{\lambda_{1}}{2 \alpha-\cos \sigma+\lambda_{1}} \simeq 2.029 \pm 0.001$.
Figure 1 resumes the variety of attractors. For $\sigma \leq 0, \quad$ feedback succeeds, $A$ diverging ( $\frac{1}{2} \cos \sigma<\alpha<\cos \sigma$ ), unless drag is too strong, bringing the bob to rest $(\alpha>\cos \sigma)$, or too weak, $A$ and $B$ diverging $\left(\alpha<\frac{1}{2} \cos \sigma\right)$. However, for $\sigma$ positive, even if small, the range $\frac{1}{2} \cos \sigma<\alpha<\cos \sigma$ exhibits bounded, nonplanar motion: oscillations with constant amplitude $\left(\alpha<\frac{1}{2} \sin \sigma+\frac{1}{2} \cos \sigma\right)$, and either oscillations of periodic amplitude or chaos $\left(\alpha>\frac{1}{2} \sin \sigma+\right.$ $\frac{1}{2} \cos \sigma$ ). 

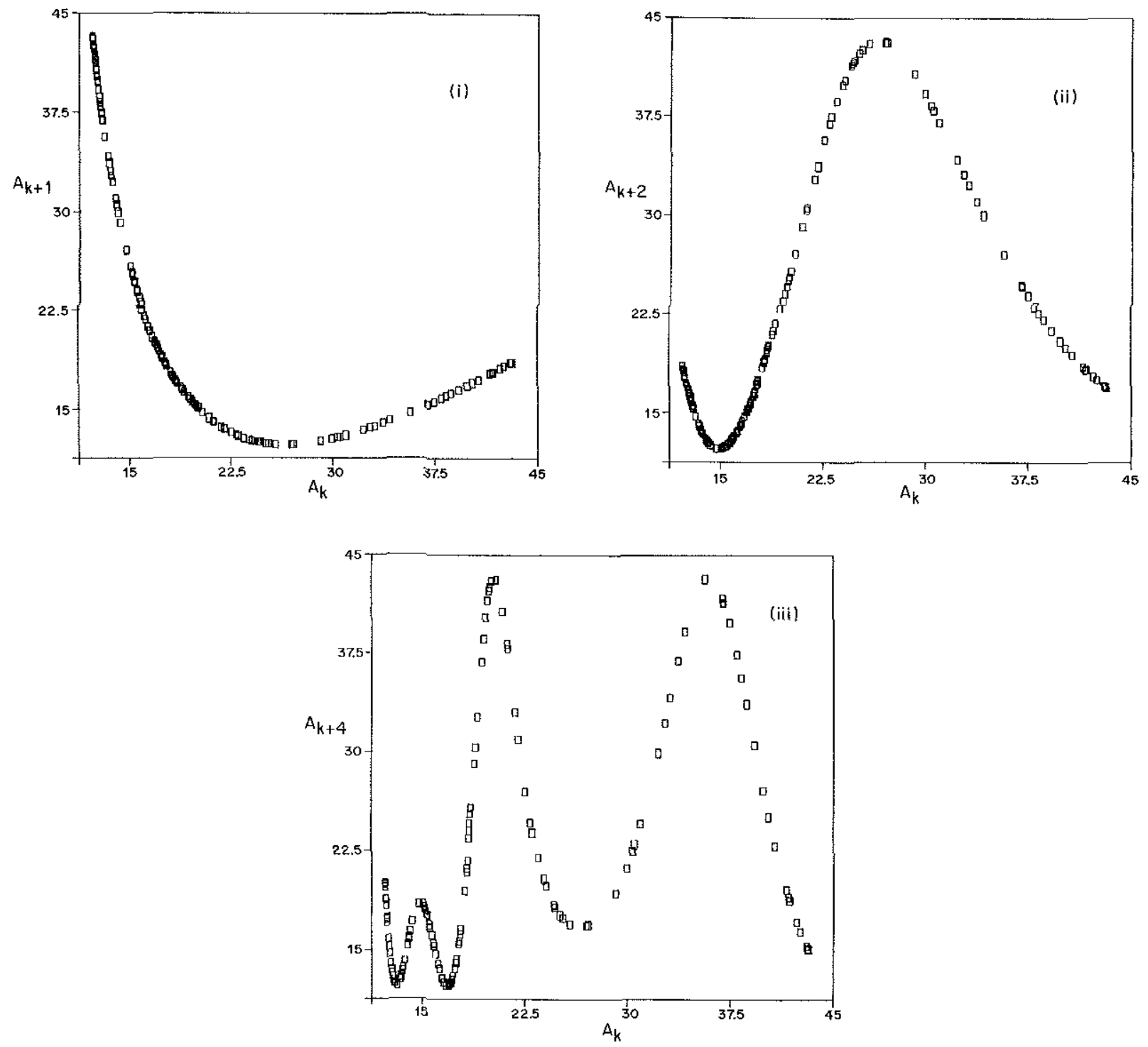

Fig. 3. (i) Approximate one-dimensional map for successive maxima of $A$ on the attractor of fig. 2c; also, maps for (ii) double, and (iii) quadruple iterates.

\section{Zero mismatch or growth rate}

We show here that bifurcations at $\sigma=0$ and $\cos \sigma-\alpha=0$ (rather than the Hopf bifurcation at $2 \alpha=\cos \sigma+\sin \sigma)$ give rise to our chaotic attractor. For the discussion to follow, it is worth recalling known features of quadratic wave interaction. For the reduced 2-wave case, there exists a time-dependent first integral at zero frequency mismatch [4] and an invariant surface at zero growth rate [5]. For the general 3-wave case, there exists a first integral if mismatch or all three growth and damping rates vanish [15]; the reduced 2-wave results may be proved to be consequences of this.

In our system growth and damping vanish jointly at $\alpha=\cos \sigma=0$ (fig. 1) and a first integral, $[A(1-\cos \gamma)-\sin \sigma] B=$ constant, then 


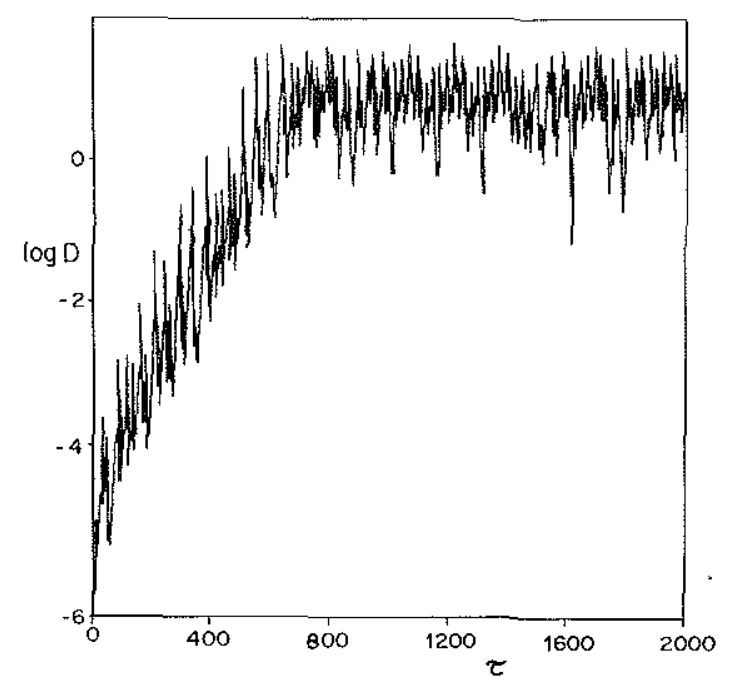

Fig. 4. Distance $D(\tau)$ between points initially close on the attractor of fig. $2 \mathrm{c}$.

exists; this family of surfaces is, however, structurally unstable. At $\alpha=\cos \sigma \neq 0(\sigma>0)$, one just has the zero-constant degenerate case: an invariant surface, $A(1-\cos \gamma)=\sin \sigma$, times the invariant plane $B=0$, producing a line of fixed points. On that plane, where $A$ is constant, there is a homoclinic orbit, $\sin \gamma=\frac{1}{2} \sin \sigma(1+\cos \gamma) \tau$, at $A=\frac{1}{2} \sin \sigma$, and periodic orbits at lower $A$; at higher $A$, two heteroclinic orbits join each $\gamma>\pi$ fixed point to its $\gamma<\pi$ symmetric point. On the invariant surface, heteroclinic orbits again map the line of fixed points into itself $(\gamma<2 \sigma$ into $\gamma>2 \sigma$ values). Only the $2 \sigma<\gamma<\pi$ fixed points are stable, point $\mathrm{P}$ of section 3 being the $2 \sigma$-end of this range; also, the invariant surface is unstable for $\gamma>\pi$.

At $\alpha=\cos \sigma=1$ (fig. 1) mismatch and growth rate vanish, no periodic orbits are left, and the invariant surface is $\gamma=0 \quad(\gamma=2 \pi)$. A dense, stable set of homoclinic orbits, $\sin \gamma=A(1-$ $\cos \gamma) \tau, A=$ constant, fill the plane $B=0$. This set is, again, structurally unstable. For $\alpha<$ $\cos \sigma=1$, there is a time-dependent first integral, $\quad A B(1-\cos \gamma) \exp (2 \alpha-1) \tau=$ constant. The generic long time behavior is $A \propto \exp (1-$ $\alpha) \tau, \gamma A \simeq 2(1-\alpha), B \propto \exp (2-3 \alpha) \tau, B$ vanishing for $\alpha>\frac{2}{3}$.
Now, the invariant surface is also structurally unstable: off condition $\alpha=\cos \sigma$, only the fixed point $\mathrm{P}$ is left. For $\alpha>\cos \sigma$, the dynamics was quite simple, $A=B=0$ being a global attractor. This referred, however, to the phase-space domain of physical solutions $A>0, B>0$, whereas $\mathrm{P}$ now lies (for $\sigma>0$ ) in the domain $A>0$, $B<0$, separated by the invariant plane $B=0$; for $\alpha-\cos \sigma \ll 1, P$ is a saddle-node near this plane with a two-dimensional stable manifold, the unstable manifold allowing both vanishing and unbounded, explosive solutions $[-B \simeq$ $\left.A \propto \gamma^{-2} \propto(\text { constant }-\tau)^{-2}\right]$. For $\alpha=\cos \sigma$, eq. (4) has two zero roots, and a negative root corresponding to the two heteroclinic orbits reaching $\mathrm{P}$ from its symmetric fixed point. For small, negative $\alpha-\cos \sigma, \mathrm{P}$ is a saddle-focus in the physical domain with a two-dimensional unstable manifold, leading to a 'screw', Rösslertype [16], chaotic attractor.

This is a hard bifurcation as regards amplitude: the full size attractor exists for vanishingly small $\cos \sigma-\alpha$ but not for $\cos \sigma-\alpha=0$, the double limit $\tau \rightarrow \infty, \alpha \rightarrow \cos \sigma$ thus being singular. Rewrite eqs. (3a,b) in terms of energy and non-driven energy fraction, and rescale appropriately:

$$
\begin{aligned}
& \tau \cos \sigma \rightarrow \tau, \quad E \equiv \frac{A+B}{\cos \sigma}, \quad \Delta \equiv \frac{B}{A+B}, \\
& \dot{E}=\left(\Delta_{*}-\Delta\right) E, \quad\left(\Delta_{*} \equiv \frac{\cos \sigma-\alpha}{\cos \sigma}\right), \\
& \dot{\Delta}=\Delta(1-\Delta)(E \sin \gamma-1) .
\end{aligned}
$$

In the physical domain $(\Delta>0)$ eqs. $(7 \mathrm{a}, \mathrm{b})$ show that $E$ will decrease without bound no matter how small $\Delta_{*}$, if negative ( $\alpha$ above $\cos \sigma$, fig. 1). At $\Delta_{*}=0$, however, $E$ decreases monotonously but remains finite; though clear from our earlier discussion, it may be shown explicitly by deriving a lower bound from $(7 \mathrm{a}, \mathrm{b})$,

$$
E(\tau>0)>E_{0}\left(1-\Delta_{0}\right)^{1 /\left(1-E_{0}\right)},
$$

with $\Delta_{0} \equiv \Delta(0)$ and $E_{0} \equiv E(0)$ assumed already 
less than unity. Similarly, the limits $\tau \rightarrow \infty$ and $\Delta_{*} \rightarrow 0^{+}(\alpha$ below $\cos \sigma)$ do not commute; no matter how small $\Delta_{*}$, if positive, there are, generically, time legs with increasing energy.

Note that the ultimate time scale for $E$ to vanish in eq. (7a) diverges as $1 /\left|\Delta_{*}\right|$ in the limit $\Delta_{*} \rightarrow 0^{-}$. For $\Delta_{*} \rightarrow 0^{+}$, the time between energy maxima on the chaotic attractor is related to the $\Delta_{*}=0$ heteroclinic structure, as shown in section 5 , and is also divergent (the bifurcation is soft as regards frequency). Thus, the limit map $E_{M^{\prime}}\left(E_{M}\right)$ relating consecutive maxima at $M$ and $M^{\prime}$ may have no Cantor structure left, and be exactly non-invertible and one-dimensional. Further, the times from $M$ to the following minimum $m$, and from $m$ to $M^{\prime}$, diverge differently; this suggests looking at $E_{M} \cdot\left(E_{M}\right)$ as a composition of partial maps $E_{m}\left(E_{M}\right), E_{M^{\prime}}\left(E_{m}\right)$. If just one partial map presents a extremum, the full map will show the unimodal signature of chaos (in the case of fig. $2 \mathrm{c}$, the map $M \rightarrow m$ is found to be monotonous).

At $\sigma=0, \frac{1}{2}<\alpha<1$ (fig. 1), there is a hard bifurcation too. For $|\sigma|$ small the fixed point $\mathrm{P}$ is again a saddle-focus with a two-dimensional unstable manifold; however, as $\sigma$ is varied from positive to negative, $\mathrm{P}$ leaves the physical domain, both $A_{\mathrm{P}}$ and $B_{\mathrm{P}}$ becoming negative. Further, the limit $\sigma \rightarrow 0, \tau \rightarrow \infty$ is also singular. As shown in section 3, for $\sigma$ negative, no matter how small, $\gamma$ decreases without bound moving periodically from $2 \pi$ to 0 , whereas for $\sigma=0, \gamma$ decreases asymptotically to zero, and for $\sigma$ positive, no matter how small, there are generically time legs with increasing $\gamma$ in eq. (3c). Finally, note that as $|\sigma| \rightarrow 0$ both $\left|A_{\mathrm{P}}\right|$ and $\left|B_{\mathrm{P}}\right|$ diverge, and so do the size of the chaotic attractor and the time between its maxima, washing out again the Cantor structure.

We rewrite eq. (3c) with the aid of an auxiliary quantity $g$,

$$
\dot{\gamma}=E \Delta(1-\cos \gamma)-\varepsilon \Delta_{*} g \quad(\varepsilon \equiv \tan \sigma),
$$

$E(1-\Delta)(1-\cos \gamma)=\varepsilon\left(1+g \Delta_{*}\right)$

$g$ is a measure of the distance to the invariant surface for $\Delta_{*}=0$, now reading $E(1-\Delta)(1-$ $\cos \gamma)=\varepsilon$, the line of fixed points being $\Delta=0$, $E(1-\cos \gamma)=\varepsilon$. In the next two sections we use eqs. $(7 \mathrm{a}-\mathrm{c})$ and $(8)$ to obtain analytical maps for the attractor. It will prove convenient to derive the equation for $\dot{g}$,

$\dot{g}=\left(1+g \Delta_{*}\right)\left(1-g \frac{\varepsilon \sin \gamma}{1-\cos \gamma}\right)$.

We note here that system $\left(3 a, b, c^{\prime}\right)$ is at variance with our system concerning the invariant surface for $\alpha=\cos \sigma$. To clarify this point, consider the system $\dot{A}=\lambda A+B f(A, \gamma)$, $\dot{B}=-\alpha B+B F(A, \gamma), \dot{\gamma}=h(A, \gamma)+B H(A, \gamma)$, which is linear in $B$. If the nonlinear terms are energy and phase-volume preserving, one must have $\partial\left(H+h_{A}\right) / \partial \gamma=0$. Now, if the stronger condition $H+h_{A}=0$ is actually satisfied, then, at vanishing linear growth rate $\lambda, h(A, \gamma)=0$ is an invariant surface of the system. For eqs. $(3 \mathrm{a}, \mathrm{b}, \mathrm{c})$ and eqs. (3a,b,c') we have $H+h_{A}=0$ and $H+$ $h_{A}=-\frac{1}{2}$, respectively.

\section{Limit map at vanishing growth rate $\left(\Delta_{*} \propto \cos \sigma-\alpha \rightarrow 0^{+}\right)$}

We analyse separately, as suggested, the two legs $M \rightarrow m, m \rightarrow M^{\prime}$ of a full $M \rightarrow M^{\prime}$ iteration. We prove that, for $\Delta_{*} \rightarrow 0^{+}$, a $M \rightarrow m$ trajectory follows a heteroclinic orbit on the $\Delta_{*}=0$ invariant surface; if $\gamma$ in the orbit reaches beyond $\pi, g$ diverges as $\Delta_{*} \rightarrow 0^{+}$(the flow being unstable on that surface) but $g \Delta_{*}$ vanishes. A $m \rightarrow M^{\prime}$ trajectory just moves up in energy, on the line of fixed points, if $\gamma_{m}<\pi$. Otherwise, the trajectory first follows a heteroclinic orbit in the plane $B=0(\Delta=0)$, from the $\gamma_{m}$ fixed point to its symmetric point; if $E_{m}>E_{\mathrm{p}}$, the motion on the line of fixed point does not occur (the motion on this line, and the beginning of the $\operatorname{leg} M \rightarrow m$, are part of the spiraling motion between the plane $B=0$ and the saddle-focus $\mathrm{P}$ that collapses into it as $\Delta_{*} \rightarrow 0^{+}$). We advance in figs. 5 i, ii a schematics of the maps derived. 

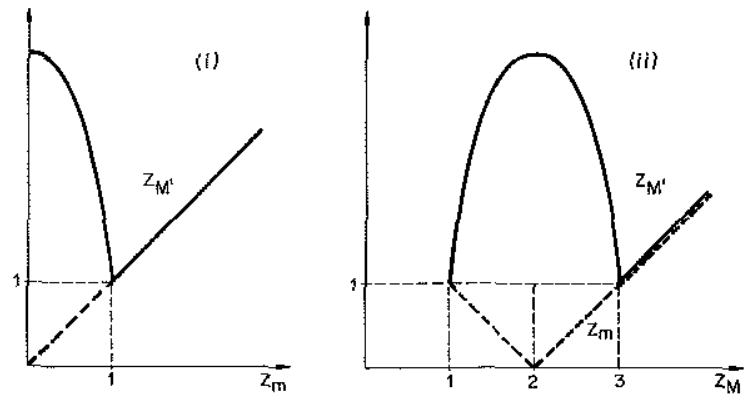

Fig. 5. Schematics of maps of extrema for the limit $\alpha-$ $\cos \sigma \rightarrow 0^{-}$: (i) partial map $m \rightarrow M^{\prime}$, (ii) partial map $M \rightarrow m(---)$, and full map $M \rightarrow M^{\prime}(-)$. $Z$ is defined in eq. (13); the peak $Z_{M^{\prime}}^{\max }$ increases with $\sigma^{-1}$.

5.1. Maximum $M\left(\Delta=\Delta_{*}, \dot{\Delta}>0\right)$ to minimum $m\left(\Delta=\Delta_{*}, \dot{\Delta}<0\right)$

Assume that (i) $\Delta_{*}$-terms in eqs. $(7 \mathrm{a}, \mathrm{c})$ may be dropped from $M$ to $m$ as $\Delta_{*}$ vanishes. Then we have

$\frac{\mathrm{d} \gamma}{\mathrm{d} E}=\cos \gamma-1 \rightarrow E-\frac{\sin \gamma}{1-\cos \gamma}=$ const. ,

and, evaluating the constant at both ends of the leg,

$E_{M}-\frac{\sin \gamma_{M}}{1-\cos \gamma_{M}}=E_{m}-\frac{\sin \gamma_{m}}{1-\cos \gamma}$.

Assume also, that (ii) $g_{M} \Delta_{*}$ and $g_{m} \Delta_{*}$ vanish in the limit $\Delta_{*} \rightarrow 0$. Then, using (8) at $M$ and $m$ yields immediately

$E_{m}\left(1-\cos \gamma_{M}\right)=\varepsilon=E_{m}\left(1-\cos \gamma_{m}\right)$.

From eqs. (11) and (12) we obtain $\gamma_{M}\left(E_{M}, \varepsilon\right)$, $\gamma_{m}\left(E_{M}, \varepsilon\right)$ and, in particular, the map for this leg, $E_{m}\left(E_{M}, \varepsilon\right)$, which takes a very simple form (fig. 5ii),

$Z_{n}=\left|Z_{M}-2\right| \quad\left(Z \equiv\left(2 \varepsilon E-\epsilon^{2}\right)^{1 / 2}\right)$,

with $Z$ as map variable; from its definition, $\varepsilon \equiv$ $\tan \sigma$ and eq. (12) we have
$E_{M} \sin \gamma_{M}=\frac{\tan \sigma}{\tan \frac{1}{2} \gamma_{M}}=Z_{M}$,
$E_{m} \sin \gamma_{m}=\frac{\tan \sigma}{\tan \frac{1}{2} \gamma_{m}}= \pm Z_{m} \quad\left(Z_{M} \lessgtr 2\right)$.

$Z_{m}=1$ corresponds to the fixed point $\mathrm{P}$ for $\Delta_{*}=$ 0 . The attractor lies in the interval $Z_{M} \geq 1 ; \gamma_{m}$, increasing with $Z_{M}$, reaches $\pi$ at $Z_{M}=2\left(Z_{m}=\right.$ $0)$.

To justify assumption (i), i.e., eq. (11), set $\Delta_{*}$ small and $Z_{M}-1=0(1)$. For the initial stage ( $\left.E \simeq E_{M}, \gamma \simeq \gamma_{M}\right)$ we use eqs. $(7 \mathrm{a}-\mathrm{c})$ and (9) to find results valid as long as $\Delta_{*} \exp \left(Z_{M}-1\right) \tau$ is small:

$\frac{\Delta}{\Delta_{*}} \simeq \exp \left(Z_{M}-1\right) \tau$,

$g \simeq\left(g_{M}-\frac{1}{Z_{M}}\right) \exp \left(-Z_{M} \tau\right)+\frac{1}{Z_{M}}$,

$\frac{E-E_{M}}{E_{M}} \simeq+\Delta_{*}\left(\frac{1-\exp \left(Z_{M}-1\right) \tau}{Z_{M}-1}+\tau\right)$,

$$
\begin{gathered}
\frac{\gamma-\gamma_{M}}{\varepsilon} \simeq-\Delta_{*}\left[\frac{1-\exp \left(Z_{M}-1\right) \tau}{Z_{M}-1}+\frac{\tau}{Z_{M}}\right. \\
\left.+\left(g_{M}-\frac{1}{Z_{M}}\right) \frac{1-\exp \left(-Z_{M} \tau\right)}{Z_{M}}\right] .
\end{gathered}
$$

Taking now $\Delta_{*} \rightarrow 0, \tau \rightarrow \infty$ in such a way that $1 \ll \exp \left(Z_{M}-1\right) \tau \ll 1 / \Delta_{*}$ yields $\Delta / \Delta_{*} \rightarrow \infty, E-$ $E_{M} \rightarrow 0$ and $\gamma-\gamma_{M} \rightarrow 0$, thus validating the lefthand side of (11); we used $g_{m} \Delta_{*} \rightarrow 0$, from assumption (ii), in eq. (14d). One similarly establishes the right-hand-side of (11) by deriving eqs. (14a-d) for the end stage $\left(E \cong E_{m}\right.$, $\gamma \simeq \gamma_{m}$ ), with $g_{m}$ instead of $g_{M}, \pm Z_{m}$ instead of $Z_{M}$ for $Z_{M} \$ 2$, and negative times $(\tau=0$ at $m$, now). Note that a term in $\gamma-\gamma_{m}$ growing as $\exp \left(-Z_{m} \tau\right)$ with negative time, for $Z_{M}<2$, is actually absent because, as shown in our discussion of assumption (ii), one has $g_{m}=1 / Z_{m}$ for such $Z_{M}$.

The end stage result is clearly valid for vanish- 
ing $Z_{m}\left(Z_{M}=2\right)$, when we have $\Delta \simeq \Delta_{*} \mathrm{e}^{-\tau}, g \simeq$ $g_{m}+\tau, \quad E-E_{m} \simeq E_{m} \Delta_{*}\left(e^{-\tau}-1+\tau\right), \quad \gamma-\gamma_{m} \simeq$ $\varepsilon \Delta_{*}\left(\mathrm{e}^{-\tau}-1+\frac{1}{2} \tau^{2}+g_{m} \tau\right)$. One may also relax the condition $Z_{M}-1=\mathscr{O}(1)$, introduced at the start. Assuming $Z_{m}-1=a \Delta_{*}^{p}=0\left(\Delta_{*}^{p}\right), 0<p<\frac{1}{2}$, eqs. (11)-(13) would give full leg changes $\left(\gamma_{m}-\right.$ $\left.\gamma_{M}\right) / \varepsilon=-\left(E_{m}-E_{M}\right) / E_{M}=\mathscr{O}\left(\Delta_{*}^{p}\right)$. Near $M$ we take $\Delta_{\psi} \rightarrow 0, \tau \rightarrow \infty$ with $1 \ll \exp \left(a \Delta_{*}^{p} \tau\right) \ll 1 / \Delta_{*}^{1-2 p}$ and find $\Delta / \Delta_{*} \rightarrow \infty,\left(\gamma-\gamma_{M}\right) /\left(\gamma_{m}-\gamma_{M}\right) \simeq(\stackrel{*}{E}-$ $\left.E_{M}\right) /\left(E_{m}-E_{M}\right) \rightarrow 0$; a similar result holds at $m$. This establishes eq. (11) for $Z_{M}-1$ vanishing with $\Delta_{*}$.

Concerning assumption (ii), we defer to section 5.2 proving that $g_{M} \rightarrow 1 / Z_{M} \leq 1$ as $\Delta_{*} \rightarrow 0$. Consider here $g_{m}$, first for $Z_{M}<2, Z_{M}-2=$ $O(1)$. Then we have $\gamma<\pi$ throughout, and eq. (9) shows that $g$ keeps values of order unity as it evolves from $1 / Z_{M}$ ( $g$ would be negative for $g>(1-\cos \gamma) / \varepsilon \sin \gamma$, which is a growing function of $\gamma$ ); further, as $\Delta$ finally decreases to again approach $\Delta_{*}$ at $m, \dot{\gamma} / \gamma$ in eq. (7c) becomes small, and then $g$ follows $\gamma$ quasisteadily, 1 $g \varepsilon \sin \gamma /(1-\cos \gamma)=0$, to yield $g_{m}=(1-$ $\left.\cos \gamma_{m}\right) / \varepsilon \sin \gamma_{m}=1 / Z_{m}$ as advanced.

For $Z_{M}>2, \gamma$ reaches beyond $\pi$, and then $g$ grows exponentially. Note that $\Delta$ in eq. (14a) would reach order unity for $\tau \sim \ln 1 / \Delta_{*}$, suggesting a leg duration of order $\ln 1 / \Delta_{k}$, to make $g_{m}$ diverge as some power of $1 / \Delta_{*}$. (For $Z_{M}=2$, the behavior found near $m, g \simeq g_{m}+\tau$, suggests that $g_{m}$ then diverges as $\left.\ln 1 / \Delta_{*}\right)$. To prove that the power is weaker than linear and thus $g_{m} \Delta_{*} \rightarrow 0$, one uses (7c), (8) and (12), dropping $\Delta_{*}$-terms, to obtain an equation for $\dot{\gamma}$ through the main stage of the leg,

$$
\dot{\gamma}=\sin \gamma-\varepsilon-\frac{\left(\sin \gamma_{M}-\varepsilon\right)(1-\cos \gamma)}{1-\cos \gamma_{M}}
$$

Matching the solution of (15) to the exact behaviors at $M$ and $m$ one determines leg duration and, using $\gamma(\tau)$ in eq. (9) with the $\Delta_{*}$-term dropped, finally determines $g_{m}$. Results are given in section 6 for the particular case of small $\varepsilon$.
5.2. Minimum $m\left(\Delta=\Delta_{*}, \dot{\Delta}<0\right)$ to following maximum $M^{\prime}\left(\Delta=\Delta_{*}, \dot{\Delta}>0\right)$

For $Z_{M}$ less than $2\left(\gamma_{m}<\pi, Z_{m}<1\right)$ and away from 1 and $2, g$ is of order unity throughout. Note that eqs. $(14 \mathrm{a}-\mathrm{d})$ with $m\left(M^{\prime}\right)$ subscripts describe the flow at the early (late) stage and, in between, when $\Delta \ll \Delta_{*}$, one has

$\dot{E} \simeq \Delta_{*} E, \quad \dot{\gamma} \simeq-\varepsilon g \Delta_{*} ;$

hence, $E$ and $\gamma$ vary slowly in a time $\tau \sim 1 / \Delta_{*}$. Since, according to (9), $g$ varies in times $\tau \sim 1$, it will follow $\gamma$ quasisteadily $[g \simeq(1-\cos \gamma) /$ $\varepsilon \sin \gamma]$, from $g_{m}=1 / Z_{m}$ to $g_{M^{\prime}}=1 / Z_{M^{\prime}}$ (as advanced) at vanishing $\Delta_{*}$, We can thus write (8) as

$E(1-\cos \gamma)=\varepsilon \quad(\sin \gamma>0)$

for the entire leg. Using (17) in the equation

$$
\begin{aligned}
& \int_{m}^{M^{\prime}}(\sin \gamma-1 / E) d E \\
& =\int_{\Delta_{*}}^{\Delta_{*}}\left(\Delta_{1}-\Delta\right) \mathrm{d} \Delta / \Delta(1-\Delta)=0,
\end{aligned}
$$

obtained from $(7 a, b)$, one gets the partial map $F_{\varepsilon}\left(Z_{M^{\prime}}\right)=F_{\varepsilon}\left(Z_{m}\right)$, where

$F_{F}(Z) \equiv Z-\frac{1}{2} \ln \left(1+Z^{2} / \varepsilon^{2}\right)-\varepsilon \tan ^{-1} Z / \varepsilon$.

The map (fig. 5i) is actually valid down to $Z_{M}=$ 1 ; as $Z_{M}$ grows from 1 to $2, Z_{m}$ moves from 1 to 0 , and $Z_{M^{\prime}}$ from 1 to $Z_{M^{\prime}}^{\max }(\varepsilon)$, given by

$F_{k}\left(Z_{M^{\prime}}^{\max }\right)=0$.

Since the duration of the leg is of order $1 / \Delta_{*}$, the minimum of $\Delta$ is exponentially small, $\ln \left(\Delta_{*} /\right.$ $\left.\Delta_{\text {min }}\right) \sim 1 / \Delta_{*}$.

For $2<Z_{M}<3, Z_{M}-2=\mathscr{O}(1)$, we again have $0<Z_{m}<1$, but now $\sin \gamma_{m}$ is negative. Since we have $g_{m} \Delta_{*} \ll 1, g$ grows exponentially in (9) at constant $\gamma$, reaching a value of order $1 / \Delta_{*}$ in a time $\sim \ln 1 / \Delta_{*}$. Then $\gamma$ itself starts to vary 
rapidly as given by (16), $\dot{\gamma}=-\varepsilon g \Delta_{*}$. Equation

(9) takes now the form

$\dot{g} \simeq \frac{-\left(1+g \Delta_{*}\right) g \varepsilon \sin \gamma}{1-\cos \gamma}$,

and thus one finds

$\frac{1-\cos \gamma}{1+\Delta_{*} g}=$ const. $=1-\cos \gamma_{m}=\frac{\varepsilon}{E_{m}}$.

As $\gamma$ decreases, $g$ grows to a maximum

$g(\gamma=\pi)=\frac{1+\cos \gamma_{m}}{1-\cos \gamma_{m}} \frac{1}{\Delta_{*}}$

and then decreases too. At a value $\gamma_{m}^{*}=2 \pi-$ $\gamma_{m}<\pi, g \Delta_{*}$ becomes small and $\gamma(\tau)$ slows down, $g$ then approaching the value $E_{m} \sin \gamma_{m}^{*}=$ $\left.E_{m} \mid \sin \gamma_{m}\right\}=Z_{m}$. Note that (a) the variation of $E$ during the $\gamma_{m} \rightarrow \gamma_{m}^{*}$ evolution vanishes with $\Delta_{*}$ $\left(E_{m}^{+}-E_{m} \rightarrow 0\right)$ and (b) the ratio $\Delta / \Delta_{*}$ at $\gamma_{m}^{*}$ is of the order of some power of $\Delta_{*}$. Since $Z_{m}$ is less than unity, we are at $\gamma_{m}^{*}$ in the same conditions prevailing in the $Z_{M}<2$ range. Thus, the leg now proceeds to a final value $Z_{M^{\prime}}$ again determined by the map $F_{\varepsilon}\left(Z_{M^{\prime}}\right)=F_{\varepsilon}\left(Z_{M}\right), \Delta / \Delta_{*}$ dropping in between to an exponentially small minimum. Clearly, the map is actually valid at $Z_{m}=2$.

For $Z_{M}>3$, finally, we again have $\sin \gamma_{m}<0, \gamma$ decreasing fast from $\gamma_{m}$ to $\gamma_{m}^{*}=2 \pi-\gamma_{m}$. Now, however, we have $Z_{m}>1$, so that $\Delta$ never gets to be exponentially small, being already increasing when $\gamma$ reaches $\gamma_{m}^{*}$. From the equation $\dot{\gamma}=\epsilon-$ $E_{m}(1-\cos \gamma)$, obtained from $\dot{\gamma}=-\varepsilon g \Delta_{*}$ and (21), one may use the inequality

$$
\int_{\gamma_{m}}^{\gamma_{m}^{*}} \frac{E_{m} \sin \gamma-1}{E_{m}(1-\cos \gamma)-\varepsilon} \mathrm{d} \gamma>0
$$

to prove $\Delta$ less than $\Delta_{*}$ when $\gamma$ reaches $\gamma_{m}^{*}$. In the limit $\Delta_{*} \rightarrow 0, \Delta$ grows then to its final value $\Delta_{*}$, at constant $E$ and $\gamma$. We thus have $Z_{m}=Z_{M^{\prime}}$, $g_{m}=g_{M^{\prime}}=1 / Z_{M^{\prime}}$.
The partial map $m \rightarrow M^{\prime}$ is therefore as shown in fig. $5 \mathrm{i}$,

$$
\begin{aligned}
& F_{\varepsilon}\left(Z_{M^{\prime}}\right)=F_{\varepsilon}\left(Z_{m}\right), \quad 0 \leq Z_{m} \leq 1, \\
& Z_{M^{\prime}}=Z_{m}, \quad Z_{m} \geq 1
\end{aligned}
$$

Figure 5ii shows both the partial map $M \rightarrow m$, eq. (13), and the full map $M \rightarrow M^{\prime}$,

$$
\begin{array}{ll}
F_{\varepsilon}\left(Z_{M^{\prime}}\right)=F_{\varepsilon}\left(\left|Z_{M}-2\right|\right), & 1<Z_{M} \leq 3, \\
Z_{M^{\prime}}=Z_{M}-2, & Z_{M} \leq 3 .
\end{array}
$$

Hughes and Proctor gave a similar map, at small growth rate, for the reduced 2-wave quadratic coupling [5]. We emphasize that (23) (a) is the exact map for the attractor of system (7), in the singular limit $\tau \rightarrow+\infty, \Delta_{*} \rightarrow 0^{+}$(which is not the attractor at $\Delta_{*}=0$ ), and (b) is valid for vanishing $Z_{M}-3$ as $\Delta_{r} \rightarrow 0$. Figure 6 compares numerical maps for $\Delta_{*}$ small with the limit form (23); the agreement is very good and improves with decreasing $\Delta_{*}$. Naturally, the iteration time diverges when approaching the limit $\Delta_{+} \rightarrow 0^{+}$.

We note here a few, simple features of (23). The maximum at $Z_{M}=2$ is mapped into the minimum at $Z_{M}=3$ for $\varepsilon$ such that $Z_{M^{\prime}}^{\max }=3$, as given by eq. (20); this occurs at $\varepsilon \equiv \tan \sigma \simeq$ 0.2031 , or $\sigma \simeq 11.48^{\circ}$. For larger $\sigma$, the attractor does not include the minimum, its map appcaring as unimodal (fig. 6i). At $\sigma=11.48^{\circ}$ there exists an infinite number of degenerate homoclinic orbits: the (maximum) critical point tends to the unstable fixed point at $Z_{M}=1$ under backward iteration, and lands on the same point under (finite) forward iteration. Below $11.48^{\circ}$, the map is bimodal (fig. 6ii), and homoclinic orbits are nondegenerate; however, for values of $\varepsilon$ in (20) such that $Z_{M^{\prime}}^{\mathrm{max}}$ is an odd integer above 3 , the map has degenerate homoclinic orbits. When $Z_{M^{\prime}}^{\max }$ in (20) is any even integer above 2, the map presents a superstable orbit; in particular, for $F_{\varepsilon}(6)=0$, i.e., $\varepsilon \approx 0.01523\left(\sigma=0.8727^{\circ}\right)$, there is a period-3 superstable orbit. 

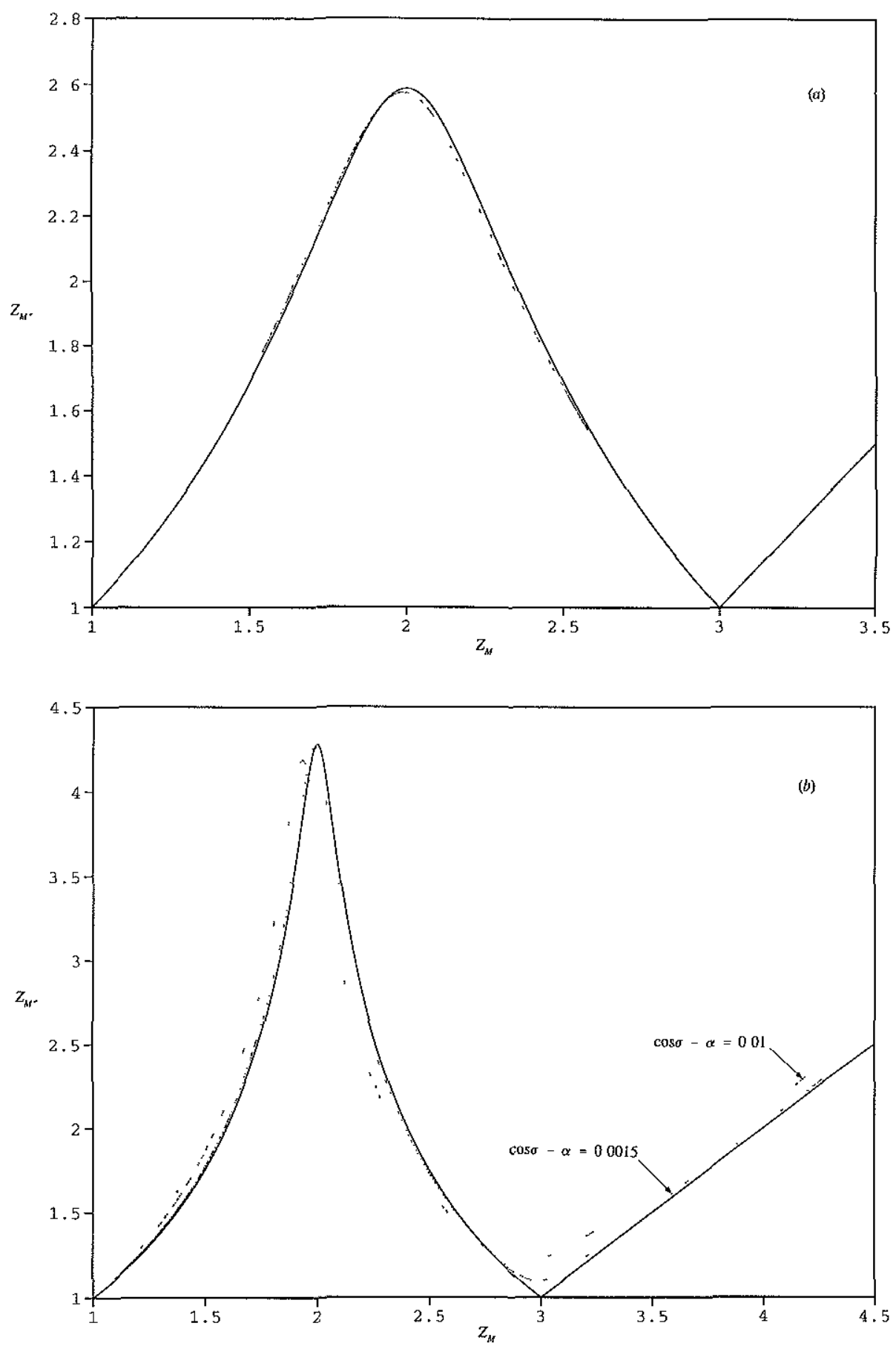

Fig. 6. (a) Map $Z_{M},\left(Z_{M}\right)$ at $\sigma=17^{\circ}$, for $\alpha-\cos \sigma \rightarrow 0^{-}$(solid line, formulae (23)), and $\alpha-\cos \sigma=0.0015$ (dots, numerical results). (b) Map at $\sigma=3.75^{\circ}$ for $\alpha-\cos \sigma \rightarrow 0^{-}$and $\alpha-\cos \sigma=0.0015,0.01$. 


\section{Limit map at ranishing phase mismatch $\left(\sigma \equiv \tan ^{-1} \varepsilon \rightarrow 0^{+}\right)$}

In the joint limit $\Delta_{*} \rightarrow 0^{+}, \varepsilon \rightarrow 0^{+}$, the map is given by eq. (23b) and by the proper limit of eq. (23a), using (19),

$$
\begin{aligned}
& Z_{M^{\prime}}-\ln Z_{M^{\prime}}=\left|Z_{M}-2\right|-\ln \left[\varepsilon^{2}+\left(Z_{M}-2\right)^{2}\right]^{1 / 2} \\
& 1 \leq Z_{M}<3
\end{aligned}
$$

The map is singular: there is a range $\left|Z_{M}-2\right|=$ $0(\varepsilon)$ for which the $\varepsilon$-term cannot be ignored no matter how small; $Z_{M^{\prime}}$ and the attractor size diverge as $\ln 1 / \varepsilon$. We now improve upon $\left(23 \mathrm{a}^{\prime}, \mathrm{b}\right)$ by considering the limit $\varepsilon \rightarrow 0^{+}$at nonvanishing $\Delta_{*}$. Lacking an explicit heteroclinic structure for that limit we restrict the study to small $\Delta_{*}(\gg \epsilon)$, keeping terms of first order. Separate analyses are required for up to eight regions in the attractor, each involving several time stages that need matching. This makes for an overlong derivation, which we omit. (It may be requested from the first author.)

For the leg $M \rightarrow m$ one must first solve for $\gamma(\tau), g(\tau)$ to lowest order in $\Delta_{*}$, as indicated in section 5 . One result is $g_{m}\left(Z_{m}, \Delta_{*}\right)$ for $Z_{M}>2$,

$$
\begin{aligned}
g_{m} & =\frac{-1}{Z_{M}-2} \\
& +\frac{4 \pi}{\left[\Delta_{*}\left(Z_{M}-2\right)^{2}\right]^{\left(Z_{M}-2\right) /\left(Z_{M}-1\right)}\left[2\left(Z_{M}-1\right)\right]^{2 /\left(Z_{M}-1\right)}} \\
& \times\left[\sin \left(\frac{\pi}{Z_{M}-1}\right)\right]^{-1}
\end{aligned}
$$

For $Z_{M} \simeq 2$ one finds independently $g_{m}=-$ $\ln \left\{\frac{1}{4} \mathrm{e} \Delta_{*}\left[\varepsilon^{2}+\left(Z_{M}-2\right)^{2}\right]^{2}\right\}$, which matches (24) smoothly in the proper limits; note that $g_{m} \Delta_{*} \rightarrow 0$ as $\Delta_{*} \rightarrow 0$ for all $Z_{m} \geq 2$, as advanced in section 5. The partial map $Z_{m}\left(Z_{M}, \Delta_{*}\right)$, for $Z_{M}<4$, is

$$
\begin{aligned}
Z_{m}= & \mid Z_{M}-2+\Delta_{*}\left\{\ln \left(\frac{4 \mathrm{e}^{2}\left(Z_{M}-1\right)^{2}}{\Delta_{*} Z_{M}^{2}}\right)\right. \\
& \left.-\frac{Z_{M}-2}{Z_{M}-1}\left[\ln \left(\frac{\left|Z_{M}-2\right|}{Z_{M}}\right)+\frac{3}{8} g_{m}^{2} \Delta_{*}\right]\right\} \mid,
\end{aligned}
$$

the underlined term being retained only for $3 \leq$ $Z_{M}<4$, when (24) is used.

The map $Z_{M}\left(Z_{m}, \Delta_{*}, \varepsilon\right)$ for the leg $m \rightarrow M^{\prime}$ reads

$$
\begin{aligned}
& Z_{M^{\prime}}-\left(1-\frac{1}{2} \Delta_{*}\right) \ln Z_{M^{\prime}} \\
& =\left.\Delta_{*} \ln \left(\frac{K_{1} I_{0}+K_{0} I_{1}}{K_{1} \pm K_{0}}\right)\right|_{Z_{m} / \Delta_{*}} \\
& -\left(1-\Delta_{*}\right) \ln \left(\varepsilon^{2}+Z_{m}^{2}\right)^{1 / 2}+\frac{1}{2} \Delta_{*} \ln \left(\frac{2 \pi}{\Delta_{*}}\right) \\
& -(1 \mp 1) \frac{\Delta_{*}}{2}\left(\frac{Z_{m}}{1+Z_{m}}+\ln \left(1+g_{m} Z_{m}\right)\right),
\end{aligned}
$$

with the upper (lower) sign used to the left (right) of the maximum of $Z_{M}$. The $g_{m}$-term only appears to the right of the maximum $\left(Z_{M}>2\right.$ to lowest order), and then $g_{m}\left(Z_{m}, \Delta_{*}\right)$ is obtained by writing $Z_{M}=2+Z_{m}$ in eq. (24). The modified Bessel functions $I_{0}, K_{0}, I_{1}, K_{1}$ are evaluated at $Z_{m} / \Delta_{*}$. One can obtain, if desired, the full map $M \rightarrow M^{\prime}$ as a single explicit expression by using $Z_{m}$ from (25) in (26), and using $g_{m}$ from (24) in both equations. This map does recover $\left(23 \mathrm{a}^{\prime}, \mathrm{b}\right)$ in the limit $\Delta_{*} \rightarrow 0$ (the limits $\varepsilon \rightarrow 0^{+}, \Delta_{*} \rightarrow 0^{+}$ do commute).

For the maximum $Z_{M^{\prime}}^{\max }\left(\Delta_{*}, \ln (1 / \varepsilon)\right)$ of the map and its abscissa $Z_{M \text { max }}\left(\Delta_{*}\right)$, and the minimum $Z_{M^{\prime}}^{\mathrm{min}}\left(\Delta_{*}\right)$ and its abscissa $Z_{M \min }\left(\Delta_{*}\right)$, we find, from (24) $-(26)$,

$$
\begin{aligned}
& Z_{M^{\prime}}^{\max }-\left(1-\frac{1}{2} \Delta_{1}\right) \ln Z_{M^{\prime}}^{\max } \\
& =\left(1-\Delta_{*}\right) \ln (1 / \varepsilon)+\frac{1}{2} \Delta_{*} \ln \left(\frac{2 \pi}{\Delta_{*}}\right), \\
& Z_{M \max }=2-\Delta_{*} \ln \left(e^{2} / \Delta_{k}\right), \\
& Z_{M^{\prime}}^{m \ln }=1+\Delta_{*}^{1 / 2}\left[\ln \left(\frac{16}{\pi^{2} e \Delta_{*}}\right)\right]^{1 / 2} \\
& +\frac{\Delta_{*}}{3}\left[\ln \left(\frac{16}{\pi^{2} e \Delta_{*}}\right)-\frac{3}{2}+\frac{3 / \pi}{\ln \left(16 / \pi^{2} e \Delta_{*}\right)}\right] \text {, } \\
& Z_{M \min }=3-\frac{3}{4} \Delta_{*}\left[\ln \left(\frac{16}{9 \Delta_{*}}\right)+5-\frac{1}{4} \pi^{2}\right] .
\end{aligned}
$$

For $\Delta_{*} \rightarrow 0$, these formulae recover results of fig. 
5 and eq. (20) (in the limit $\varepsilon \rightarrow 0$ ). Since $Z_{M^{\prime}}^{\min }-$ $1 \sim \Delta_{*}^{1 / 2}\left(\ln \left(1 / \Delta_{*}\right)\right)^{1 / 2} \gg \Delta_{*}^{1 / 2}$, one has $Z_{M}-1 \gg$ $\Delta_{*}^{1 / 2}$ throughout the attractor; this confirms our discussion on the value $Z_{M}=1$ in section 5.1 (it also avoids a singularity in the first logarithm of (25)). Figure 7 shows very good agreement between (28)-(30) and numerical results for $\varepsilon=$ $0.01<\Delta_{*}$; at such small $\varepsilon$ the accurate determination of $Z_{M^{\prime}}^{\max }$ takes a very long computing time.

As suggested by fig. 7 , the minimum at $Z_{M \min }$ is mapped into the maximum at $Z_{M \max }$ at some finite value $\bar{\Delta}_{*}, Z_{M \max }\left(\bar{\Delta}_{*}\right)=Z_{M^{\prime}}^{\min }\left(\bar{\Delta}_{*}\right)$; the approximate use of $(28),(29)$ yields $\bar{\Delta}_{*} \simeq 0.129$. For $\Delta_{*}>\bar{\Delta}_{*}$ the attractor excludes the maximum, as it excluded the minimum for $\varepsilon>0.2031$ $\left(\Delta_{*}=0\right)$ : the map is bimodal only inside a domain by the corner $\varepsilon=0, \Delta_{*}=0$. The case of fig. 2c $\left(\varepsilon \simeq 0.06554, \Delta_{*} \simeq 0.1782\right)$ lies outside. Comparing the numerical results for this case with present theoretical results for $\varepsilon=0^{+}$and $\Delta_{*}$ very small gives a $2-10 \%$, still reasonable, disagreement.

\section{Summary of results}

A spherical swing is an example of damped, nonlinearly coupled oscillators with a $1: 1$ frequency ratio, one oscillator being driven coherently for efficient excitation. We explored here whether, and how, a phase-mismatch between drive and response might make this response chaotic. Our model involves parameters for damping and mismatch. We found that for certain damping range the excitation succeeds if it lags behind, but may produce a chaotic attractor if it leads the response.

Although the attractor may be reached through a Feigenbaum sequence as damping increases, it is actually born elsewhere. A hard (as regards amplitude) bifurcation occurs at the zero growth-rate parametric line, as damping decreases, when an unstable fixed point crosses an invariant plane to enter as saddle-focus a phase-space domain of physical solutions. A second hard bifurcation occurs at the zero-mis-

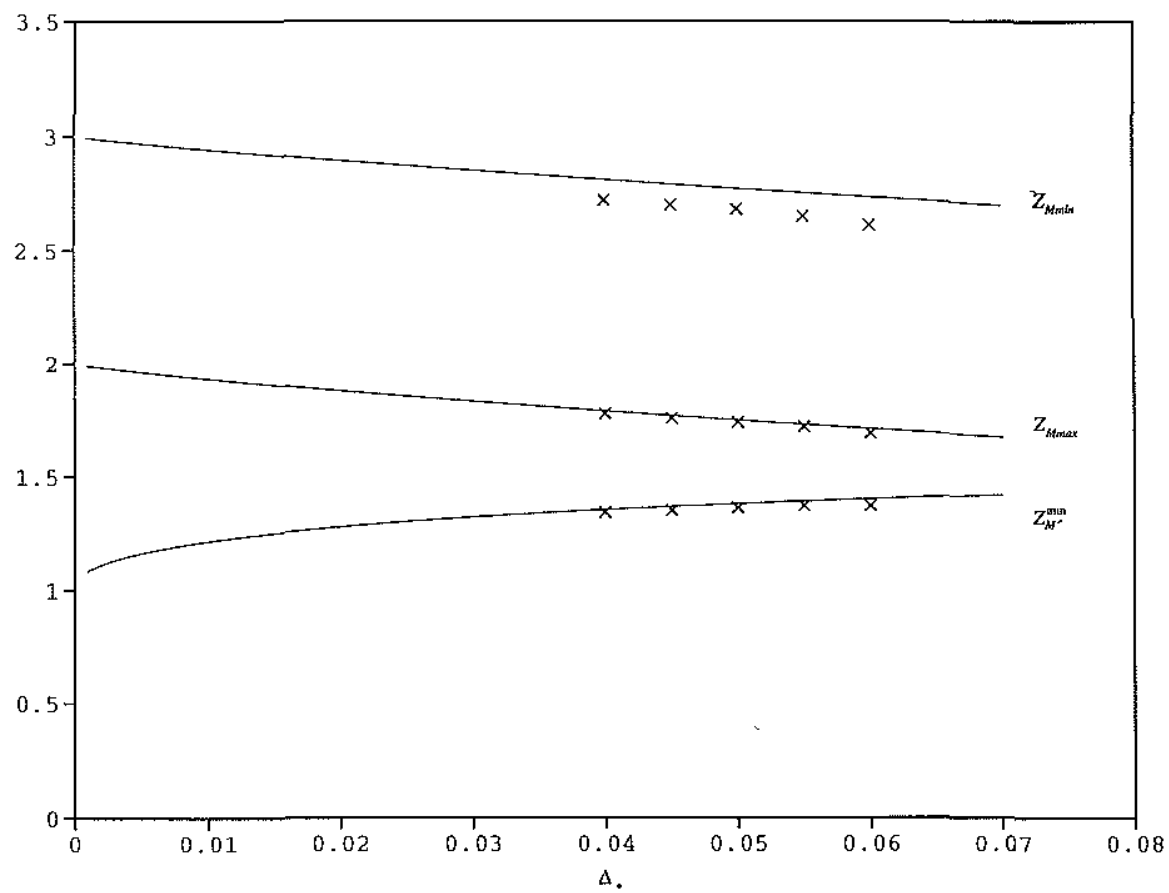

Fig. 7. Coordinates of extrema in the map $Z_{M} \cdot\left(Z_{M}\right)$ vs $\Delta_{*} \equiv(\cos \sigma-\alpha) / \cos \sigma$, for $\epsilon \equiv \tan \sigma=0.01$. Formulae (28)-(30), ——; numerical results, $x$. 
match line, when the saddle-focus leaves that domain. Times on the attractor diverge as one approaches either line, resulting on exactly onedimensional and non-invertible (no Cantor structure) limit maps, which are bimodal near the corner of those lines. A complex heteroclinic structure and a line of fixed points existing at zero growth-rate allow to analytically determine such maps; this effectively represents an exact solution of the chaotic dynamics of the differential equations [17].

Work in progress concerns mismatched coherent driving for general cubic coupling, and other frequency ratios. Tentative results, yet to be published, suggest that some of the features found here may be generic. This might be useful for a broader problem closely related to ours, wave interaction in a nonlinear medium, which is the basis of current work on spatio-temporal chaos [18].

\section{Acknowledgements}

We are grateful to Prof. C.F. Dewey for encouragement in this work, which was supported in part by the US National Institute of Health, Grant No. HL 25536, and the Comision Interministerial de Ciencia y Technologia of Spain (PB 85 59). One author (J.R.S.) acknowledges support from the Fluid Mechanics Laboratory during a stay there.

\section{References}

[1] J.B. McLaughlin, J. Stat. Phys. 24 (1981) 375;

D. D'Humieres, M.R. Beasley, B.A. Huberman and A. Libchaber, Phys. Rev. A 26 (1982) 3483;

A.H. MacDonald and M. Plischke, Phys. Rev. B 27 (1983) 201;

B.P. Koch and R.W. Leven, Physica D 16 (1985) 1.

[2] P.J. Holmes and D.A. Rand, Q. Appl. Math 35 (1978) 495;
Y. Ueda and N. Akamatsu, IEEE Trans. Circuits Syst. CAS 28 (1980) 217;

M. Levi, Mem. Am. Math. Soc. 214 (1981) 1;

R. Shaw, Z. Naturforsch. 36a (1981) 80.

[3] P.J. Holmes and D.C. Whitley, Physica D 7 (1983) 111; R. Raty, J. van Boehm and H.M. Isömaki, Phys. Lett. A 103 (1984) 289;

U. Parlitz and W. Lauterborn, Phys. Lett. A 107 (1985) 351 ;

R. Seydel, Physica D 17 (1985) 308.

[4] J.M. Wersinger, J.M. Finn and E. Ott, Phys. Fluids 23 (1980) 1142;

M.N. Bussac, Phys. Rev. Lett. 49 (1982) 1939.

[5] D.W. Hughes and M.R.E. Proctor, Physica D 46 (1990) 163.

[6] D.A. Russell and E. Ott, Phys. Fluids 24 (1981) 1976.

[7] J. Guckenheimer and A. Mahalov, Physica D 54 (1992) 267.

[8] J.R. Sanmartín, Am. J. Phys. 52 (1984) 937;

J.V. Breakwell and J.W. Gearhart, J. Astron. Sci. 35 (1987) 19;

M. Martinez-Sanchez and S.A. Gavit, AlAA J. Guidance 10 (1987) 233.

[9] J. Miles, Physica D 11 (1984) 309; see also Quart. Appl. Math. 20 (1962) 21.

[10] D.J. Tritton, Eur. J. Phys. 7 (1986) 162;

F.E. Irons, Eur. J. Phys. 11 (1990) 107.

[11] E. Ott, C. Grebogi and J.A. Yorke, in: Chaos/Xaoc, D.K. Campbell, ed. (American Institute of Physics, New York, 1990) pp. 153-172;

R.H. Abraham, in: A Chaotic Hierarchy, G. Baier and M. Klein, eds. (World Scientific, Singapore, 1991) pp. 49-78.

[12] J.R. Sanmartín, in: Synergetics, Order and Chaos, M.G. Velarde, ed. (World Scientific, Singapore, 1988) pp. 55-65.

[13] B.D. Hassard, N.D. Kazarinoff and Y-H. Wan, Theory and Applications of Hopf Bifurcation (Cambridge University, Cambridge, 1981) pp. 86-91.

[14] D.A. Russell, J.D. Hanson and E. Ott, Phys. Rev. Lett. 45 (1980) 1175.

[15] B. Coppi, M.N. Rosenbluth and R.N. Sudan, Ann. Phys. (NY) 55 (1969) 207;

R.C. Davidson, Methods in Nonlinear Plasma Theory (Academic Press, New York, 1972) § 6.4.4;

$\mathrm{J}$. Weiland and H. Wilhelmsson, Coherent Nonlinear Interaction of Waves in Plasmas (Pergamon, Oxford, 1977) Chs. 7, 9, 10 and 12.

[16] O. Rössler, Ann. NY Acad. Sci. 316 (1979) 376.

[17] A.C. Fowler and M.J. McGuinness, Physica D 5 (1982) 149.

[18] C.C. Chow, A. Bers and A.K. Ram, Plasma Phys. Control. Fusion 34 (1992) 1945. 